\title{
Yokukansan for perioperative psychiatric symptoms in cancer patients undergoing high invasive surgery. J-SUPPORT 1605 (ProD Study): study protocol for a randomized controlled trial
}

\author{
Saho Wada ${ }^{1,2}$, Ryoichi Sadahiro ${ }^{1,2}$, Yutaka J. Matsuoka ${ }^{1,2,3}$, Yosuke Uchitomi ${ }^{1,3,4}$, Takuhiro Yamaguchi ${ }^{5}$
} and Ken Shimizu, ${ }^{1,23^{*}}$ (D)

\begin{abstract}
Background: Preoperative anxiety and postoperative delirium affect both short- and long-term prognoses in patients with cancer; therefore, these conditions require early prevention and treatment. However, no standard preventive or therapeutic methods have been established for them. Yokukansan, a Japanese herbal medicine for the treatment of insomnia and anxiety, causes relatively few adverse drug reactions and effectively improves the behavioral and psychological symptoms of dementia. Thus, it is expected to be useful for treating and/or preventing perioperative psychiatric symptoms in patients with cancer. The objective of this study is to clarify the therapeutic effect of Yokukansan for preoperative anxiety and its preventive effect on postoperative delirium in cancer patients, as well as to confirm its safety profile.

Methods: This study is a randomized, double-blind, placebo-controlled study in cancer patients scheduled to undergo tumor resection. Patients who provide consent are randomly allocated to receive oral administration of Yokukansan or placebo, and study drug administration is continued for 4 days or longer prior to surgery. We defined two primary endpoints, change in preoperative anxiety and incidence of postoperative delirium. Secondary endpoints are severity score of postoperative delirium, duration of postoperative delirium, amount of benzodiazepines used prior to surgery, amount of antipsychotic agents used after surgery, and number of postoperative hospitalization days. We plan to complete the analysis on March 31, 2021. The target number of registered patients is 110 per group, or 220 in total.

Discussion: This study is the first randomized, double-blind, placebo-controlled study intended to clarify the effects of a Japanese herbal medicine, Yokukansan, in the prevention and treatment of perioperative psychiatric symptoms in patients with cancer. The trial was initiated on August 14, 2017, with 195 subjects randomized by October 5, 2018.
\end{abstract}

Trial registration: UMIN Clinical Trials Registry (UMIN-CTR), UMIN000027561. Registered on 31 May 2017. Keywords: Yokukansan, Oncology, Surgery, Anxiety, Delirium

\footnotetext{
* Correspondence: keshimiz@ncc.go.jp

'Department of Psycho-Oncology, National Cancer Center Hospital, 5-1-1,

Tsukiji, Chuo-ku, Tokyo, Japan

${ }^{2}$ Division of Health Care Research, Behavioral Sciences and Survivorship

Research Group, Center for Public Health Sciences, National Cancer Center

Japan, 5-1-1, Tsukiji, Chuo-ku, Tokyo, Japan

Full list of author information is available at the end of the article
}

(c) The Author(s). 2019 Open Access This article is distributed under the terms of the Creative Commons Attribution 4.0 International License (http://creativecommons.org/licenses/by/4.0/), which permits unrestricted use, distribution, and reproduction in any medium, provided you give appropriate credit to the original author(s) and the source, provide a link to the Creative Commons license, and indicate if changes were made. The Creative Commons Public Domain Dedication waiver (http://creativecommons.org/publicdomain/zero/1.0/) applies to the data made available in this article, unless otherwise stated. 


\section{Background}

Patients with cancer experience various psychiatric symptoms during the perioperative period. Anxiety not only lowers treatment compliance by impairing cancer patients' decision-making ability [1], but it also exacerbates cancer symptoms, leading to reduced quality of life [2]. During the perioperative period, anxiety has also been reported to prolong postoperative pain [3], affect endocrine and immune functions, and influence the progression and long-term prognosis of cancer [4]. An observational study previously conducted at our hospital showed that preoperative anxiety occurred in approximately $15 \%$ of cancer patients [5]. Hence, we consider that reducing preoperative anxiety is an important goal of cancer treatment in general.

Delirium is a psychiatric disorder characterized by acute impairment of consciousness and lack of attention [6]. Postoperative delirium refers to delirium that occurs after patients recover from anesthesia, typically within 2 or 3 days after the operation [7-10]. Patients with postoperative delirium experience not only increased rates of respiratory infection and complications associated with immobility and prolongation of hospitalization, but also long-term adverse effects including increased mortality rate [11-13] and impaired cognitive function [14-16]. In the aforementioned observational study, postoperative delirium occurred in approximately $32 \%$ of cancer patients [5].

Perioperative psychiatric symptoms should be appropriately assessed, treated, and prevented. However, when used in patients with impaired metabolic capacity, benzodiazepines, which are common therapeutic agents for anxiety, may have long-lasting effects or cause muscle relaxation, resulting in falls. They are also considered to induce delirium when used in patients with cerebral vulnerability, including elderly patients and patients with dementia [17-21]. The antipsychotic agents used for the treatment of postoperative delirium may cause dose-dependent over-sedation or serious adverse drug reactions that include extrapyramidal symptoms and long QT syndrome. Moreover, antipsychotic agents have been reported to increase mortality risk when used in elderly patients with dementia and are often difficult to use in the treatment of elderly patients or patients with heart disease [22-24].

In these contexts, Yokukansan has attracted attention for its potential utility in the treatment of perioperative psychiatric symptoms in patients with cancer. Yokukansan is a Japanese herbal medicine for the treatment of insomnia and anxiety, and it effectively improves the behavioral and psychological symptoms of dementia (BPSD), including hallucinations, delusions, disinhibition, day/night reversal, wandering, irritability, verbal aggression, and risky behavior [25-29]. It has been hypothesized that Yokukansan may prevent postoperative delirium, which presents clinical symptoms very similar to those of BPSD [27, 30-34].

Several lines of empirical evidence imply that Yokukansan would be effective in this context. In a retrospective study $(n=19)$ in patients who underwent colorectal surgery, we found that the incidence of postoperative delirium after Yokukansan administration was lower than in previous studies [35]. In a pre/post study of patients who underwent gastrointestinal surgery $(n=$ 8 ), oral administration of Yokukansan improved insomnia, dysesthesia, restless legs and behavior, and risky behavior [31]. In a randomized phase II trial in which patients underwent cardiovascular surgery $(n=30)$, Yokukansan significantly improved agitation, mood swings, deficient sense of reality, hallucinations, and delusions [33]. In another randomized phase II trial in patients undergoing surgery for gastrointestinal and lung malignancies $(n=186)$ [36], Yokukansan decreased the risk of postoperative delirium in patients with a Mini Mental State Examination score $\leq 26$.

Adverse drug reactions, including hypokalemia, have been observed following the use of Yokukansan, but their incidence was no higher than 1.3\% (41 of 3141 patients) $[37,38]$. On the other hand, benzodiazepines and antipsychotic agents commonly used for these symptoms can cause serious adverse reactions. Although the effect of Yokukansan on anxiety and delirium is less certain than that of benzodiazepines and antipsychotic agents, major adverse events have not been observed following Yokukansan administration, suggesting that this herbal medication may have a major advantage in regard to safety.

Against this background, we planned a randomized controlled study on the efficacy of Yokukansan for perioperative psychiatric symptoms. The objective of this study is to clarify the therapeutic effect of Yokukansan for preoperative anxiety, its preventive effect on postoperative delirium, and its safety in patients with cancer.

\section{Methods \\ Study design}

The therapeutic effect of Yokukansan for preoperative anxiety, its preventive effect on postoperative delirium, and its safety in cancer patients will be confirmed in a randomized, double-blind, placebo-controlled study. The investigators registered this study in the UMIN Clinical Trials Registry (UMIN-CTR) https://upload.umin.ac.jp/ cgi-open-bin/ctr/ctr_view.cgi?recptno=R000031488

(UMIN000027561) prior to the start of the trial.

\section{Study setting and sample}

We are conducting our study at the National Cancer Center Hospital, a tertiary hospital for cancer in Japan. 
We are recruiting patients meeting all of the following inclusion criteria: (1) histologically confirmed malignant tumor or clinically confirmed neoplastic lesion that is speculated to be appropriately treatable by resection; (2) scheduled to undergo highly invasive surgery, defined as tumor resection estimated to last $6 \mathrm{~h}$ or longer [39]; (3) Hospital Anxiety and Depression Scale-Anxiety (HADS-A) score of at least 1 at the time of registration; (4) ability to take study drugs orally; and (5) age of 20 years or older.

Exclusion criteria are as follows: (1) diagnosis of delirium based on the Diagnostic and Statistical Manual of Mental Disorders, Fifth Edition (DSM-5) at the time of recruitment; (2) use of any Japanese herbal medicine within 4 weeks before the day of registration; (3) history of allergy to Japanese herbal medicine; and (4) hypokalemia rated as grade 2 or higher according to the Common Terminology Criteria for Adverse Events, fourth version (CTCAE v 4.0) within 8 weeks before the day of registration.

The subject accrual period is from August 2017 to March 2019, and we plan to complete the analysis by March 31, 2021.

\section{Registration and randomization}

The investigators are conducting a screening survey of patients scheduled to undergo highly invasive surgery, as specified in the inclusion criteria. The outline of this study is shown in Figs. 1 and 2. The Standard Protocol Items: Recommendations for Interventional Trials (SPIRIT) checklist is provided as Additional file 1.

Because the drugs are indistinguishable in appearance, the study is blinded to the person responsible for study drug allocation. Eligibility is confirmed at the time of registration, and treatment groups are randomly allocated. No stratification factors are used. The investigators are not informed in detail about the random allocation procedures. The subject registration and random allocation of treatment groups are conducted by a Web-based system. Randomization was balanced with randomly permuted blocks and implemented with an interactive Web-response system which assigned a unique code that dictated the treatment assignment and matching study drug kit for each patient. Thus, treatment assignments were masked from patients and study personnel. Prior to data analysis, the allocation table is retained by the person responsible for studying drug allocation. We have not set criteria for discontinuing or modifying allocated interventions for a given subject.

\section{Intervention}

From the day the surgery date was determined to the day before surgery, one packet of the study drug is administered orally three times a day, before or between meals. The intervention group receives Tsumura Yokukansan Extract Granules (TJ-54, Tsumura \& Co., Tokyo, Japan). The daily dose of $7.5 \mathrm{~g}$ contains the following herbal medicines: Atractylodes Lancea Rhizome 19.5\%, Poria Sclerotium 19.5\%, Cnidium Rhizome 14.6\%, Uncaria Hook 14.6\%, Japanese Angelica Root 14.6\%, Bupleurum Root 9.7\%, and Glycyrrhiza 7.3\%. The placebo group receives granules prepared from lactose and other ingredients not containing Yokukansan extract powder, with an appearance and taste formulated to be as similar as possible to those of Yokukansan.

The treatment period is specified as 4 days or longer prior to the surgery. Compliance is rated on the following 3-point scale: (1) at least two-thirds of the dose was taken, (2) at least one-third but less than two-thirds of the dose was taken, and (3) less than one-third of the dose was taken. We prohibit the use of glycyrrhizin and other herbal medicines for 4 weeks before administration of the study drugs.

The HADS-A is used to assess anxiety before and after administration. Subsequently, delirium is assessed daily using the DSM- 5 from 1 to 5 days postoperatively. In subjects with delirium only, the severity of delirium is rated using the Delirium Rating Scale-Revised-98 (DRS-R-98), and the duration of delirium is assessed until it resolves or until 30 days after surgery, whichever is later.

\section{Primary outcomes}

Change in preoperative anxiety (change in HADS-A score)

Preoperative anxiety is assessed using the HADS-A [40, 41]. The HADS is a self-reported measure of mental status related to depression and anxiety and consists of 14 questions in total: seven pertain to depression (HADS-D), and the remaining seven are about anxiety (HADS-A). Subjects are asked to respond to HADS-A questions on a 4-point scale from 0 to 3; thus, the maximum score is 21 . A higher score indicates worse anxiety. In this study, the efficacy of the study drug is assessed by the difference in the HADS-A score before and after oral administration. Researchers ask for the HADS-A results by phone before Yokukansan or placebo is administered for the first time, and they assess the second HADS-A score in the afternoon the day before surgery.

\section{Incidence of postoperative delirium}

The proportion of patients diagnosed with postoperative delirium is calculated among those in whom planned surgery is completed. In this study, trained psychiatrists or clinical psychologists assess postoperative delirium on a daily basis using DSM-5 [6] within 5 days after surgery. The DSM-5 offers a standard criterion for the classification of mental disorders. Many mental health professionals use the manual to determine a patient's diagnosis and to communicate this diagnosis to other practitioners. 


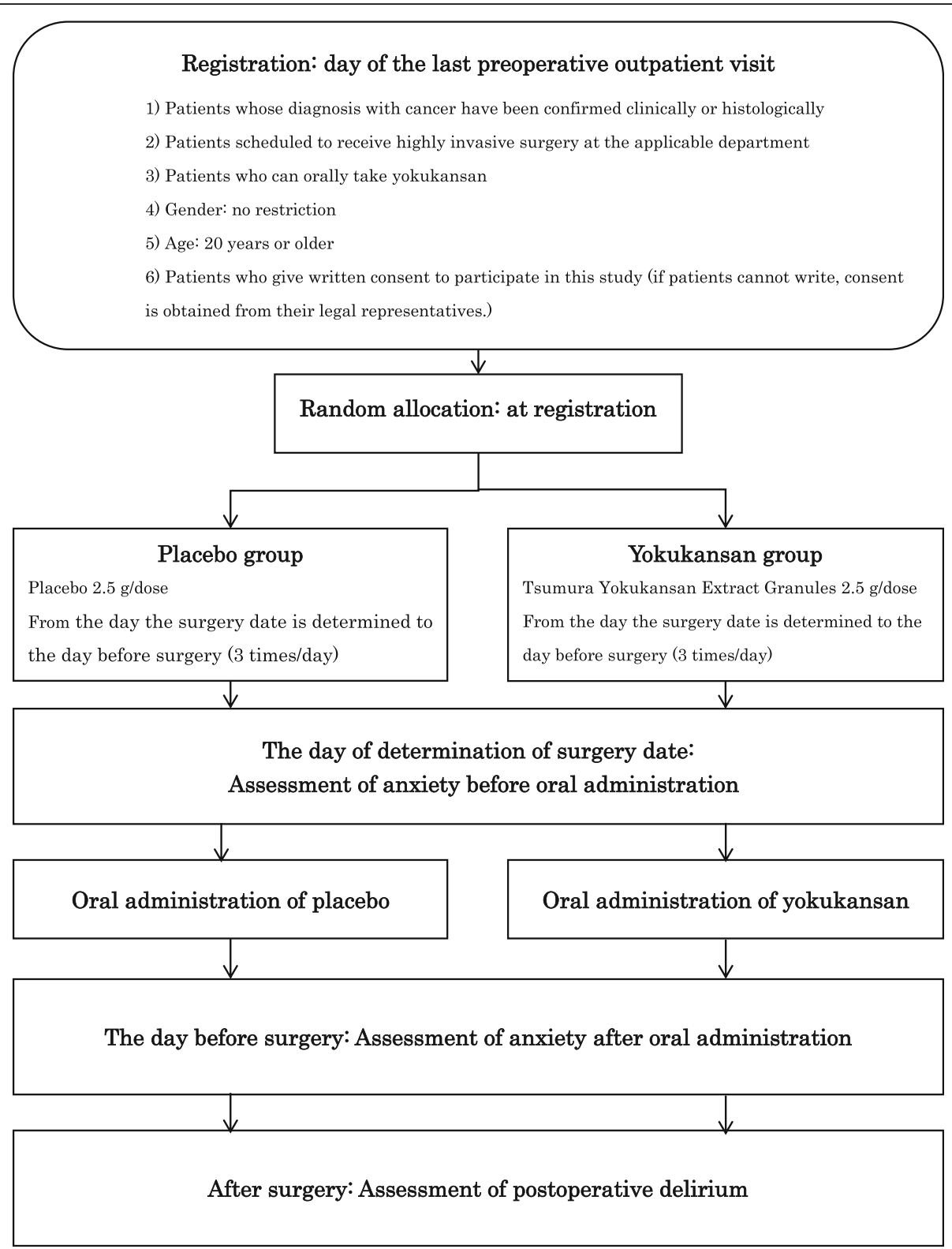

Fig. 1 Flowchart of the study procedure

We clinically demonstrated and discussed our findings on postoperative delirium before this study, with a final determination made once there was satisfactory agreement between the researchers and the principal investigator (SW) regarding the diagnosis of delirium. The researchers assessed patient samples simultaneously and independently to check the inter-rater reliability of the DSM-5 (20 times for each researcher).

\section{Secondary outcomes}

The secondary outcomes are severity score of postoperative delirium assessed by duration of postoperative delirium, amount of benzodiazepines used before surgery, amount of antipsychotic agents used after surgery, and number of days of postoperative hospitalization. The severity of delirium is assessed using the Japanese version of DRS-R-98 [42, 43]. Although this scale rates delirium using the sum of two subscales-the severity score, based on the evaluation of 13 items, and the scores for three items related to diagnosis-this study used only the severity score. Each item is scored from 0 to 2 or 0 to 3, with a higher score indicating a more severe condition.

\section{Safety outcomes}

We define adverse reactions as any undesirable or unintended signs, symptoms, or diseases that occur from the 


\begin{tabular}{|c|c|c|c|c|c|}
\hline & \multicolumn{5}{|c|}{ STUDY PERIOD } \\
\hline & \multirow{2}{*}{$\begin{array}{c}\begin{array}{c}\text { Enrollment } \\
\& \\
\text { Allocation }\end{array} \\
\text { T0 }\end{array}$} & \multicolumn{4}{|c|}{ Post-allocation } \\
\hline TIMEPOINT ${ }^{* *}$ & & $\begin{array}{c}4-8 \\
\text { preoperative } \\
\text { days } \\
\text { (T1) } \\
\end{array}$ & $\begin{array}{c}1 \\
\text { preoperative } \\
\text { day } \\
\text { (T2) } \\
\end{array}$ & $\begin{array}{c}1-5 \\
\text { postoperative } \\
\text { days } \\
\text { (T3) } \\
\end{array}$ & $\begin{array}{c}30 \\
\text { postoperative } \\
\text { days } \\
\text { (T4) }\end{array}$ \\
\hline \multicolumn{6}{|l|}{ ENROLLMENT: } \\
\hline \multirow{2}{*}{$\begin{array}{l}\text { Eligibility screen } \\
\text { Informed consent }\end{array}$} & $\mathrm{X}$ & & & & \\
\hline & $\mathrm{X}$ & & & & \\
\hline Allocation & $x$ & & & & \\
\hline \multicolumn{6}{|l|}{ INTERVENTIONS: } \\
\hline \multicolumn{6}{|l|}{ Placebo } \\
\hline \multicolumn{6}{|l|}{ Yokukansan } \\
\hline \multicolumn{6}{|l|}{ ASSESSMENTS: } \\
\hline \multirow{3}{*}{$\begin{array}{r}\text { Demographic } \\
\text { Preoperative } \\
\text { anxiety: } \\
\text { HADS-A } \\
\text { Postoperative } \\
\text { delirium: } \\
\text { DSM-5, DRS-98-R, } \\
\text { duration }\end{array}$} & $\mathrm{x}$ & & & & \\
\hline & & $x$ & $\mathrm{x}$ & & \\
\hline & & & & & \\
\hline \multicolumn{6}{|l|}{ BZPs use } \\
\hline \multicolumn{6}{|l|}{$\begin{array}{l}\text { Antipsychotic } \\
\text { agents use }\end{array}$} \\
\hline \multicolumn{6}{|l|}{$\begin{array}{l}\text { Days of postoperat } \\
\text { ive hospitalization }\end{array}$} \\
\hline Adverse events & & & & & \\
\hline
\end{tabular}

Fig. 2 SPIRIT figure: schedule of enrollment, interventions, and assessments. HADS-A Hospital Anxiety and Depression Scale-Anxiety, DSM-5 Diagnostic and Statistical Manual of Mental Disorders, Fifth Edition, DRS-R-98 Delirium Rating Scale-Revised-98, BZP benzodiazepine

day a study drug is administered through 5 days after surgery. We do not consider events to be adverse reactions if they are judged by the participating surgeons to be related to the surgery.

We describe the details of serious adverse events. The number of cases and the rate are calculated for each study group based on the CTCAE v 4.0. Regarding clinical laboratory values, we calculate summary statistics of changes from actual values and pre-dose values for each treatment group.

\section{Sample size calculation}

In this study, two primary endpoints are specified. If the superiority of Yokukansan over placebo for either endpoint is confirmed, the drug would be considered to be effective. Therefore, considering the Bonferroni adjustment for multiplicity, a two-sided significance level of $2.5 \%$ is used for each endpoint. Regarding the incidence of postoperative delirium-one of the primary endpoints-a previous meta-analysis reported that antipsychotics reduced the incidence of delirium by $50 \%$ compared to placebo [44]. The effect of Yokukansan against BPSD, which is thought to be a condition similar to delirium, has repeatedly been demonstrated in randomized controlled trials and is non-inferior to that of antipsychotics [27, 30-34], so we anticipated obtaining the same results against postoperative delirium. Therefore, we estimated an effect size for Yokukansan of 0.5 for postoperative delirium. Yokukansan has few side effects, and if we can show that its effects are comparable to those of antipsychotics, the results may change current clinical practice, in which antipsychotics are generally used as a first line pharmacotherapy to prevent postoperative delirium. Based on an observational study that we performed using exactly the same inclusion and exclusion criteria at the same hospital [5], we estimated 
the incidence of delirium at $40 \%$ in the placebo group and $20 \%$ in the Yokukansan group. Given a two-sided significance level of $2.5 \%$ and a statistical power of $80 \%$, this indicates that 99 subjects per group are necessary. As for the change in preoperative anxiety, the minimum clinically meaningful difference in anxiety score was shown in a previous study to be 1.32 points, although in that case the subjects were patients with chronic obstructive lung disease [45]. Therefore, in this study we assume a between-group difference in the mean change in preoperative anxiety of 1.5 points. Assuming a 3 -point standard deviation in the preoperative anxiety score, a two-sided significance level of $2.5 \%$, and a statistical power of $80 \%$ based on the results of an observational study conducted at the National Cancer Center Hospital [13], 78 subjects per group are necessary. Considering the above and speculating that some subjects may later be found to be ineligible or may withdraw from the study, the planned number of registered subjects was determined to be 110 per group, or 220 in total.

\section{Statistical analysis}

The analysis sets for efficacy and safety include all registered subjects. The mean change in the HADS-A score before and after oral administration in each group, the point estimate of the between-group difference, and the 97.5\% confidence interval are calculated. Group means are compared using the $t$ test. The incidence of postoperative delirium in each group, the point estimate of the between-group difference, and the $97.5 \%$ confidence interval are calculated, and the proportions are compared by the chi-square test. Because there are two primary endpoints, Bonferroni adjustment for multiplicity is performed, and a two-sided significance level of $2.5 \%$ is used for the two comparisons. If superiority of the Yokukansan group is observed in either of the endpoints, treatment with Yokukansan will be considered to be useful. The interim analysis examined only the Yokukansan group but not both groups, and we do not regard this as a multiple analysis.

Interim analysis will be performed when the number of subjects who have completed the protocol treatment and are evaluable for both endpoints reaches one-third $(n=74)$ of 220 , which is the target number of registered patients in this study. At that time, we will determine whether or not continuation of the study is appropriate. If it is determined that the main purpose of the study has not been fulfilled, the study will be discontinued, and the study results will be promptly disclosed. In principle, registration will not be suspended during the interim analysis. When the point estimate of the mean preoperative anxiety score in the Yokukansan group exceeds 5 points or the incidence of postoperative delirium exceeds $40 \%$ in the interim analysis, the Independent Data Monitoring Committee, which consists of three external members, will judge whether or not to discontinue the study.

Serious adverse events are described in detail. For other adverse events, the number of subjects with the highest observed grade and the incidence of the highest grade in each treatment group are calculated, and the groups are compared by the Mantel test.

We define the analysis sets as follows. The full analysis set consists of all randomized subjects who satisfy eligibility criteria, receive any study drug, participate in at least one postbaseline assessment, and have no Good Clinical Practice (GCP) violations. The per-protocol set excludes subjects in which the study is not carried out as per protocol because consent is withdrawn, whose surgery is canceled, who do not take the study drug according to the protocol, etc. The safety analysis set includes all subjects who receive any study treatment (including control) but excludes subjects who withdraw prior to receiving any treatment. Data that have been rejected due to missing information, lack of clarity, or faulty handling of data are not included in the analysis. We do not impute the missing data.

\section{Quality control and assurance}

The study-related division of the sponsor, Tsumura \& Co., performs quality control. Clinical research associates decide on issues related to data entered into the electronic data capture system and report their conclusions to the investigator. If any problems are anticipated, the investigator takes the appropriate measures.

To determine whether the study is being conducted in accordance with the protocol and the GCP, the GCP audit division of the sponsor performs a GCP audit and formulates an appropriate plan following the TsumuraGCP audit procedure.

The GCP audit division of the sponsor performs GCP audits of all facilities involved in this study, including the study site and facilities at external organizations and contract research organizations, at appropriate times during or after the study.

\section{Research ethics approval}

This study was designed and is undertaken in compliance with the Declaration of Helsinki and the "Ethical Guidelines for Medical and Health Research Involving Human Subjects" (Public Notice of the Ministry of Education, Culture, Sports, Science and Technology and the Ministry of Health, Labor and Welfare). All study protocols, informed consent forms, and other requested documents were approved by the Institutional Review Board and the Ethics Committee of the National Cancer Center in Japan. 
The investigators sufficiently explain the details of the study to the subjects in accordance with the informed consent documents and obtain subjects' voluntary written consent to participate in the study. After provision of consent, subjects are withdrawn from the study according to the following criteria: (1) if the subject withdraws consent; (2) if the primary disease is exacerbated or an adverse event occurs, and continuation of the study is judged to be difficult; (3) if a subject is found to be ineligible for the study after it begins; and (4) if a subject discontinues the study treatment at his or her own discretion.

The documents or records that should be stored at the study site, including those related to subject consent, those on which case report forms are based, records of deliberation by the review board, documents related to requests and contracts for the study, and the management table for study drugs, will be stored for 3 years after the discontinuation or completion of the study. After the storage period, the documents and records will be anonymized and discarded by an appropriate method. We do not plan to grant public access to the full protocol, participant-level dataset, or statistical code.

\section{Funding source and conflicts of interest in this study}

Funding and other resources necessary for the conduct of this study are provided by Tsumura \& Co., the sponsor. In conducting and reporting this study, the investigator and coinvestigator must not change their professional judgment for the purpose of obtaining monetary profit or other personal benefits. The investigator and coinvestigators are not related to Tsumura \& Co. by employment or personal connections. If patent or related rights arise in association with this study, the study site and the sponsor determine the appropriate right-holders.

The sponsor also provides study drugs and prepares study-related materials, including the protocol and statistical analysis plan, and supports the preparation of the informed consent documents and the form for withdrawal of consent.

The principal investigator is responsible for writing the report and deciding whether to publish it.

\section{Discussion}

Preoperative anxiety and postoperative delirium affect both short- and long-term prognoses in patients with cancer and therefore need to be appropriately assessed, treated, and prevented. However, neither preventive nor therapeutic methods have been established for these conditions.

The present study is the first randomized, double-blind, placebo-controlled study conducted to clarify the effects of a Japanese herbal medicine, Yokukansan, for the prevention and treatment of perioperative psychiatric symptoms in patients with cancer. Although Yokukansan has already been approved for anxiety, existing findings are insufficient to allow a clear conclusion regarding its efficacy in preoperative conditions in particular. There are no standard therapeutic regimens for preoperative anxiety or postoperative delirium in cancer patients. For these reasons, if the present study can clarify the therapeutic effect of Yokukansan on preoperative anxiety, its preventive effect on postoperative delirium, and its safety in cancer patients, it will greatly contribute to the establishment of standard therapies for perioperative psychiatric symptoms in this population, leading to the provision of safer medical care with higher compliance. However, if the effect of Yokukansan is not verified in this study, the lack of clear evidence will suggest that continued use of this agent in daily practice can be stopped.

\section{Trial status}

The protocol version number is Ver 3.2, and the date is September 6, 2017. The trial was initiated on August 14, 2017, with 195 subjects randomized by October 5, 2018. We plan to complete recruitment on March 31, 2019.

\section{Additional file}

Additional file 1: SPIRIT 2013 checklist: recommended items to address in a clinical trial protocol and related documents. (DOC $124 \mathrm{~kb}$ )

\section{Abbreviations \\ PSD: Behavioral and psychological symptoms of dementia; CTCAE $\vee$ 4.0: Common Terminology Criteria for Adverse Events, fourth version; DRS-R- 98: Delirium Rating Scale-Revised-98; DSM-5: Diagnostic and Statistical Manual of Mental Disorders, Fifth Edition; GCP: Good Clinical Practice; HADS- A: Hospital Anxiety and Depression Scale-Anxiety; HADS-D: Hospital Anxiety and Depression Scale-Depression; J-SUPPORT: Japan Supportive, Palliative and Psychosocial Oncology Group}

\section{Acknowledgements}

The authors thank Asao Ogawa, M.D., Ph.D., Sadamoto Zenda, M.D., Ph.D., and Nobuhiro Sugano, M.D., Ph.D. for their advising on planning the study. The authors also express special gratitude to Yumi Kikukawa, CP and Hiroko Takagi, PhC for their help in conducting the study, and all staff that cooperated with this study.

This study is reviewed by Japan Supportive, Palliative and Psychosocial Oncology Group (J-SUPPORT) in terms of the adequacy of the research involved, and approved as J-SUPPORT study 1605.

Funding

Funding and other resources necessary for the conduct of this study are provided by Tsumura \& Co.

\section{Availability of data and materials}

The data that support the findings of this study are available from Tsumura $\&$ Co., but restrictions apply to the availability of these data, which were used under license for the current study, and so are not publicly available. Data are however available from the authors upon reasonable request and with permission of Tsumura \& Co.

Authors' contributions

SW is the principal investigator. SW and KS conceived the study and drafted the original protocol. TY is responsible for the statistical aspects of the trial including sample size calculation and statistical analysis plan. SW, KS, RS, YJM, 
and YU participated in the refinements of the protocol. SW, KS, and RS managed the experiment. SW managed the research assistants. SW and KS managed the enrollment procedure and overall regulation of the trial. All authors read and approved the final manuscript.

\section{Ethics approval and consent to participate}

Informed written consent was obtained from all participants. The Ethical Review Board, National Cancer Center Japan approved the study protocol.

\section{Consent for publication}

\section{Not applicable.}

\section{Competing interests}

Dr. Shimizu has received a Grant-in-Aid for Scientific Research (C) from the Japan Society for the Promotion of Science (15K08832), from Research for Promotion of Cancer Control Programmes (H27-political-general), and from Tsumura \& Co. Dr. Sadahiro has received Sasakawa Foundation Butterfield awards (B108) and a grant from SGH Foundation. Dr. Matsuoka has received a Grant-in-Aid for Scientific Research (B) from the Japan Society for the Promotion of Science (17H04253), an intramural research grant for Neurological and Psychiatric Disorders from the National Center of Neurology and Psychiatry Japan (27-3-2), and a grant from SENSHIN Medical Research Foundation, and has also received speaker's honoraria from Eli Lilly Japan K.K., Morinaga Milk Industry Co., Ltd., and Takeda Pharmaceutical Company Ltd. Dr. Uchitomi has received Grants from the National Cancer Center Research and Development Fund (27-A-3), Research for Promotion of Cancer Control Programmes (H29-political-general), and Japan Agency for Medical Research and development, AMED. Dr. Yamaguchi received consulting fees from Tsumura \& Co.

\section{Publisher's Note}

Springer Nature remains neutral with regard to jurisdictional claims in published maps and institutional affiliations.

\section{Author details}

'Department of Psycho-Oncology, National Cancer Center Hospital, 5-1-1, Tsukiji, Chuo-ku, Tokyo, Japan. ${ }^{2}$ Division of Health Care Research, Behavioral Sciences and Survivorship Research Group, Center for Public Health Sciences, National Cancer Center Japan, 5-1-1, Tsukiji, Chuo-ku, Tokyo, Japan. ${ }^{3}$ Innovation Center for Supportive, Palliative and Psychosocial Care, National Cancer Center Hospital, 5-1-1, Tsukiji, Chuo-ku, Tokyo, Japan. ${ }^{4}$ Behavioral Sciences and Survivorship Research Group, Center for Public Health Sciences, National Cancer Center Japan, 5-1-1, Tsukiji, Chuo-ku, Tokyo, Japan. ${ }^{5}$ Division of Biostatistics, Tohoku University Graduate School of Medicine, 1-1 Seiryo-machi, Aoba-ku, Sendai, Miyagi 980-8574, Japan.

Received: 29 March 2018 Accepted: 16 January 2019 Published online: 08 February 2019

\section{References}

1. El Jawahri A, Traeger L, Park E, Greer J, Pirl W, Lennes I, Jackson V, Gallagher E, Temel J. Associations among prognostic understanding, quality of life, and mood in patients with advanced cancer. Cancer. 2014;120:278-85.

2. Koizumi A, Matsushima E, Mochizuki Y, Omura K, Amagasa T. Changes in the psychological characteristics of oral cancer patients in the perioperative period: a quantitative evaluation. J Med Dent Sci. 2013;60:41-53.

3. Aigner CJ, Hernandez M, Koyyalagunta L, Novy D. The association of presurgery psychological symptoms with postsurgery pain among cancer patients receiving implantable devices for pain management. Support Care Cancer. 2014;22:2323-8.

4. Horowitz M, Neeman E, Sharon E, Ben-Eliyahu S. Exploiting the critical perioperative period to improve long-term cancer outcomes. Nat Rev Clin Oncol. 2015;12:213-26

5. Wada S, Inoguchi H, Sadahiro R, Matsuoka YJ, Uchitomi Y, Sato T, Shimada K, Yoshimoto S, Daiko H, Shimizu K. Preoperative anxiety as a predictor of delirium in cancer patients: a prospective observational cohort study. World J Surg. 2019;43:134-42.

6. Takahashi S, Oono Y. Desk reference to the diagnostic criteria from DSM-5. Philadelphia: American Psychiatric Association; 2014.
7. Gallagher TK, McErlean S, O'Farrell A, Hoti E, Maguire D, Traynor OJ, Conlon KC, Geoghegan JG. Incidence and risk factors of delirium in patients post pancreaticoduodenectomy. HPB (Oxford). 2014;16:864-9.

8. Takeuchi M, Takeuchi H, Fujisawa D, Miyajima K, Yoshimura K, Hashiguchi S, Ozawa S, Ando N, Shirahase J, Kitagawa Y, Mimura M. Incidence and risk factors of postoperative delirium in patients with esophageal cancer. Ann Surg Oncol. 2012;19:3963-70.

9. Yamagata K, Onizawa K, Yusa H, Wakatsuki T, Yanagawa T, Yoshida H. Risk factors for postoperative delirium in patients undergoing head and neck cancer surgery. Int J Oral Maxillofac Surg. 2005;34:33-6.

10. Yoshimura Y, Kubo S, Shirata K, Hirohashi K, Tanaka H, Shuto T, Takemura S, Kinoshita $\mathrm{H}$. Risk factors for postoperative delirium after liver resection for hepatocellular carcinoma. World J Surg. 2004;28:982-6.

11. Ely EW, Shintani A, Truman B, Speroff T, Gordon SM, Harrell FE Jr, Inouye SK, Bernard GR, Dittus RS. Delirium as a predictor of mortality in mechanically ventilated patients in the intensive care unit. JAMA. 2004;291:1753-62.

12. Pisani MA, Kong SY, Kasl SV, Murphy TE, Araujo KL, Van Ness PH. Days of delirium are associated with 1-year mortality in an older intensive care unit population. Am J Respir Crit Care Med. 2009;180:1092-7.

13. van den Boogaard M, Schoonhoven L, van der Hoeven JG, van Achterberg T, Pickkers P. Incidence and short-term consequences of delirium in critically ill patients: a prospective observational cohort study. Int J Nurs Stud. 2012; 49:775-83.

14. Girard TD, Jackson JC, Pandharipande PP, Pun BT, Thompson JL, Shintani AK, Gordon SM, Canonico AE, Dittus RS, Bernard GR, Ely EW. Delirium as a predictor of long-term cognitive impairment in survivors of critical illness. Crit Care Med. 2010;38:1513-20.

15. Pandharipande PP, Girard TD, Jackson JC, Morandi A, Thompson JL, Pun BT, Brummel NE, Hughes CG, Vasilevskis EE, Shintani AK, et al. Long-term cognitive impairment after critical illness. N Engl J Med. 2013;369:1306-16.

16. Saczynski JS, Inouye SK, Kosar CM, Tommet D, Marcantonio ER, Fong T, Hshieh T, Vasunilashorn S, Metzger ED, Schmitt E, et al. Cognitive and brain reserve and the risk of postoperative delirium in older patients: analysis of data from a prospective observational study. Lancet Psychiatry. 2014;1:437-43.

17. Inouye SK, Westendorp RG, Saczynski JS. Delirium in elderly people. Lancet. 2014;383:911-22.

18. Kalisvaart KJ, Vreeswijk R, de Jonghe JF, van der Ploeg T, van Gool WA, Eikelenboom P. Risk factors and prediction of postoperative delirium in elderly hip-surgery patients: implementation and validation of a medical risk factor model. J Am Geriatr Soc. 2006;54:817-22.

19. Robinson TN, Raeburn CD, Tran ZV, Angles EM, Brenner LA, Moss M Postoperative delirium in the elderly: risk factors and outcomes. Ann Surg. 2009;249:173-8.

20. van Meenen LC, van Meenen DM, de Rooij SE, ter Riet G. Risk prediction models for postoperative delirium: a systematic review and meta-analysis. J Am Geriatr Soc. 2014;62:2383-90

21. Litaker D, Locala J, Franco K, Bronson DL, Tannous Z. Preoperative risk factors for postoperative delirium. Gen Hosp Psychiatry. 2001;23:84-9.

22. Gill SS, Bronskill SE, Normand SL, Anderson GM, Sykora K, Lam K, Bell CM, Lee PE, Fischer HD, Herrmann N, et al. Antipsychotic drug use and mortality in older adults with dementia. Ann Intern Med. 2007;146:775-86.

23. Kishi T, Hirota T, Matsunaga S, Iwata N. Antipsychotic medications for the treatment of delirium: a systematic review and meta-analysis of randomised controlled trials. J Neurol Neurosurg Psychiatry. 2016;87:767-74.

24. Schneeweiss S, Setoguchi S, Brookhart A, Dormuth C, Wang PS. Risk of death associated with the use of conventional versus atypical antipsychotic drugs among elderly patients. CMAJ. 2007;176:627-32.

25. Hayashi $Y$, Ishida $Y$, Inoue $T$, Udagawa $M$, Takeuchi $K$, Yoshimuta $H$, Kiue K, Ninomiya Y, Kawano J, Sameshima T, et al. Treatment of behavioral and psychological symptoms of Alzheimer-type dementia with Yokukansan in clinical practice. Prog Neuro-Psychopharmacol Biol Psychiatry. 2010;34:541-5.

26. Iwasaki K, Kosaka K, Mori H, Okitsu R, Furukawa K, Manabe Y, Yoshita M, Kanamori A, Ito N, Wada K, et al. Improvement in delusions and hallucinations in patients with dementia with Lewy bodies upon administration of yokukansan, a traditional Japanese medicine. Psychogeriatrics. 2012;12:235-41.

27. Matsuda $Y$, Kishi T, Shibayama H, Iwata N. Yokukansan in the treatment of behavioral and psychological symptoms of dementia: a systematic review and meta-analysis of randomized controlled trials. Hum Psychopharmacol. 2013;28:80-6. 
28. Mizukami K, Asada T, Kinoshita T, Tanaka K, Sonohara K, Nakai R, Yamaguchi K, Hanyu H, Kanaya K, Takao T, et al. A randomized cross-over study of a traditional Japanese medicine (kampo), yokukansan, in the treatment of the behavioural and psychological symptoms of dementia. Int Neuropsychopharmacol. 2009;12:191-9.

29. Nagata K, Yokoyama E, Yamazaki T, Takano D, Maeda T, Takahashi S, Terayama Y. Effects of yokukansan on behavioral and psychological symptoms of vascular dementia: an open-label trial. Phytomedicine. 2012; 19:524-8.

30. Furukawa K, Tomita N, Uematsu D, Okahara K, Shimada H, Ikeda M, Matsui T, Kozaki K, Fujii M, Ogawa T, et al. Randomized double-blind placebocontrolled multicenter trial of Yokukansan for neuropsychiatric symptoms in Alzheimer's disease. Geriatr Gerontol Int. 2017;17:211-8.

31. Kurauchi N, Mukai N, Yoshida A. Initial trial of administration of Yokukansan for postoperative delirium. Hakodateigakushi. 2009:33:1-4

32. Monji A, Takita M, Samejima T, Takaishi T, Hashimoto K, Matsunaga H, Oda M, Sumida Y, Mizoguchi Y, Kato T, et al. Effect of yokukansan on the behavioral and psychological symptoms of dementia in elderly patients with Alzheimer's disease. Prog Neuro-Psychopharmacol Biol Psychiatry. 2009;33:308-11.

33. Takase S, Yokoyama S. Shuujutsukiniokeru kanpo no ouyou. koureisha sinnzoudaikekkannshujutu jutugosenmou ni taisuru Yokukansan no yoboukouka. Kanpo to saishintiryo. 2013;22:113-9.

34. Tsurumaki T, Terumitsu M, Yamada Y. A case report; preoperative administration of Yokukansan might have preventive effect of postoperative delirium. Nihonshikamasuigakkaizasshi. 2014;42:636-7.

35. Wada S, Inoguchi H, Hirayama T, Matsuoka YJ, Uchitomi Y, Ochiai H, Tsukamoto S, Shida D, Kanemitsu Y, Shimizu K. Yokukansan for the treatment of preoperative anxiety and postoperative delirium in colorectal cancer patients: a retrospective study. Jpn J Clin Oncol. 2017;47:844-8.

36. Sugano N, Aoyama T, Sato T, Kamiya M, Amano S, Yamamoto N, Nagashima T, Ishikawa Y, Masudo K, Taguri M, et al. Randomized phase II study of TJ-54 (Yokukansan) for postoperative delirium in gastrointestinal and lung malignancy patients. Mol Clin Oncol. 2017;7:569-73.

37. Hisada T, Maki A, Katori M. Yakuzai no Rinsho Yokukansan no fukusayou hatusgenhindo chosa. Shindan to Chiryo. 2016;104:640-7.

38. Arai YC, Kawanishi J, Sakakima Y, Sueoka S, Ito A, Tawada Y, Maruyama Y, Banno S, Takayama $H$, Nishihara $M$, et al. The effect of the kampo medicine yokukansan on preoperative anxiety and sedation levels. Evid Based Complement Alternat Med. 2014;2014:965045.

39. Shah S, Weed HG, He X, Agrawal A, Ozer E, Schuller DE. Alcohol-related predictors of delirium after major head and neck cancer surgery. Arch Otolaryngol Head Neck Surg. 2012;138:266-71.

40. Bjelland I, Dahl AA, Haug TT, Neckelmann D. The validity of the Hospital Anxiety and Depression Scale. An updated literature review. J Psychosom Res. 2002;52:69-77.

41. Kugaya A, Akechi T, Okuyama T, Okamura H, Uchitomi Y. Screening for psychological distress in Japanese cancer patients. Jpn J Clin Oncol. 1998;28:333-8.

42. Kato M, Kishi Y, Okuyama T, Trzepacz PT, Hosaka T. Japanese version of the Delirium Rating Scale, Revised-98 (DRS-R98-J): reliability and validity. Psychosomatics. 2010;51:425-31.

43. Trzepacz PT, Mittal D, Torres R, Kanary K, Norton J, Jimerson N. Validation of the Delirium Rating Scale-revised-98: comparison with the delirium rating scale and the cognitive test for delirium. J Neuropsychiatry Clin Neurosci. 2001;13:229-42.

44. Hirota T, Kishi T. Prophylactic antipsychotic use for postoperative delirium: a systematic review and meta-analysis. J Clin Psychiatry. 2013;74:e1136-44.

45. Puhan MA, Frey M, Buchi S, Schunemann HJ. The minimal important difference of the hospital anxiety and depression scale in patients with chronic obstructive pulmonary disease. Health Qual Life Outcomes. 2008;6:46.

\section{Ready to submit your research? Choose BMC and benefit from}

- fast, convenient online submission

- thorough peer review by experienced researchers in your field

- rapid publication on acceptance

- support for research data, including large and complex data types

- gold Open Access which fosters wider collaboration and increased citations

- maximum visibility for your research: over $100 \mathrm{M}$ website views per year

At BMC, research is always in progress.

Learn more biomedcentral.com/submissions 